Fixed Point Theory, 20(2019), No. 2, 559-566

DOI: $10.24193 /$ fpt-ro.2019.2.36

http://www.math.ubbcluj.ro/ nodeacj/sfptcj.html

\title{
FIXED POINT RESULTS IN B-METRIC SPACE
}

\author{
ZORAN D. MITROVIĆ \\ Nonlinear Analysis Research Group \\ Ton Duc Thang University \\ Ho Chi Minh City, Vietnam \\ and \\ Faculty of Mathematics and Statistics \\ Ton Duc Thang University \\ Ho Chi Minh City, Vietnam \\ E-mail: zoran.mitrovic@tdtu.edu.vn
}

\begin{abstract}
We give another proof of Czerwik's fixed point theorem in the setting of b-metric spaces, improving a recent version of this theorem in b-metric spaces obtained in [M. Jovanović, Z. Kadelburg, S. Radenović, Common fixed point results in metric-type spaces, Fixed Point Theory Appl., (2010)]. An analogue of Reich contraction principle and Kannan's fixed point theorem is proved in this space. Our results generalize many known results in fixed point theory.
\end{abstract}

Key Words and Phrases: Fixed points, b-metric space.

2010 Mathematics Subject Classification: 47H10, 54H25.

Acknowledgement. The author is grateful to the anonymous reviewer at careful check of details and useful comments who have improved this work. The author is also grateful to Professor Stojan Radenović for his help.

\section{REFERENCES}

[1] H. Aydi, M.F. Bota, E. Karapinar, S. Mitrović, A fixed point theorem for set-valued quasicontractions in b-metric spaces, Fixed Point Theory Appl., (2012), 2012:88.

[2] I.A. Bakhtin, The contraction mapping principle in quasimetric spaces, Funct. Anal., Ulianowsk Gos. Ped. Inst., 30(1989), 26-37.

[3] V. Berinde, Generalized contractions in quasimetric spaces, Seminar on Fixed Point Theory, (1993), 3-9.

[4] M. Bota, A. Molnár, C. Varga, On Ekeland's variational principle in b-metric spaces, Fixed Point Theory, 12(2011), 21-28.

[5] M. Cicchese, Questioni di completezza e contrazioni in spazi metrici generalizzati, Boll. Un. Mat. Ital., 5(1976), 175-179.

[6] M. Cosentino, P. Salimi, P. Vetro, Fixed point results on metric-type spaces, Acta Math. Sci. Ser. B Engl. Ed., 34(2014), no. 4, 1237-1253.

[7] S. Czerwik, Contraction mappings in b-metric spaces, Acta Math. Inform. Univ. Ostrava, 1(1993), 5-11.

[8] S. Czerwik, Nonlinear setvalued contraction mappings in b-metric spaces, Atti Sem. Mat. Univ. Modena, 46(1998), 263-276. 
[9] M.B. Jleli, B. Samet, C. Vetro, F. Vetro, Fixed points for multivalued mappings in b-metric spaces, Abstr. Appl. Anal., (2015), Art. ID 718074, 7 pages.

[10] M. Jovanović, Z. Kadelburg, S. Radenović, Common fixed point results in metric-type spaces, Fixed Point Theory Appl., (2010), Art. ID 978121, 15 pages.

[11] Z. Kadelburg, S. Radenovic, Pata-type common fixed point results in b-metric and b-rectangular metric spaces, J. Nonlinear Sci. Appl., 8(2015), 944-954.

[12] R. Kannan, Some results on fixed points, Bull. Calcutta Math. Soc., 60(1968), 71-76.

[13] M.A. Khamsi, Remarks on cone metric spaces and fixed point theorems of contractive mappings, Fixed Point Theory Appl., (2010), Art. ID 315398, 7 pages.

[14] M.A. Khamsi, N. Hussain, KKM mappings in metric type spaces, Nonlinear Anal., 73(2010), 3123-3129.

[15] M. Kir, H. Kizitune, On some well known fixed point theorems in b-metric spaces, Turk. J. Anal. Numb. Theory, 1(2013), 13-16.

[16] W. Kirk, N. Shahzad, Fixed Point Theory in Distance Spaces, Springer, Berlin, 2014, pp. 113131.

[17] P.K. Mishra, S. Sachdeva, S.K. Banerjee, Some fixed point theorems in b-metric space, Turk. J. Anal. Numb. Theory, 2(2014), 19-22.

[18] S.K. Mohanta, Some fixed point theorems using wt-distance in b-metric spaces, Fasc. Math., 54(2015), 125-140.

[19] S. Reich, Some remarks concerning contraction mappings, Canad. Math. Bull., 14(1971), 121124.

Received: January 4, 2017; Accepted: April 27, 2018. 